\title{
openheart Icosapent ethyl: scientific and legal controversies
}

\author{
Gregory Curfman (D) , ${ }^{1}$ Emile Shehada ${ }^{2}$
}

To cite: Curfman G, Shehada E. Icosapent ethyl: scientific and legal controversies. Open Heart 2021;8:e001616. doi:10.1136/ openhrt-2021-001616

Accepted 1 April 2021

Check for updates

(C) Author(s) (or their employer(s)) 2021. Re-use permitted under CC BY-NC. No commercial re-use. See rights and permissions. Published by BMJ.

1JAMA, American Medical Association, Chicago, Illinois, USA

${ }^{2}$ Yale University Law School, New Haven, Connecticut, USA

Correspondence to Dr Gregory Curfman; Gregory. curfman@jamanetwork.org

\section{ABSTRACT}

Icosapent ethyl (Vascepa) is a purified preparation of the omega-3 fatty acid eicosapentaenoic acid, which is marketed by Amarin Pharma based in Ireland. The product was initially approved by the US Food and Drug Administration for the use of a high dose ( $4 \mathrm{~g} /$ day) in the treatment of hypertriglyceridaemia. On the basis of the results of the REDUCE-IT (Reduction of Cardiovascular Events with Icosapent Ethyl Intervention Trial), the agency later granted a label extension to include the additional indication of a reduction in risk of cardiovascular events in persons with serum triglyceride levels of $150 \mathrm{mg} /$ $\mathrm{dL}$ or greater and established cardiovascular disease or diabetes. Data supporting the efficacy of omega-3 fatty acids in the prevention of cardiovascular disease have been inconsistent and controversial. The story of the development of icosapent ethyl has been fraught with challenges, including the invalidation of six core patents on the product, and recently, the completion of a new clinical trial, STRENGTH (Long-Term Outcomes Study to Assess STatin Residual Risk Reduction With EpaNova in HiGh CV Risk PatienTs With Hypertriglyceridemia), that directly contradicts REDUCE-IT and calls into question whether icosapent ethyl is actually effective in the secondary prevention of cardiovascular events. This article traces the course of the development of this fascinating product and discusses its complex medical, regulatory and legal history, which is still continuing to unfold.

When in March 2020 six core patents issued for the omega-3 fatty acid marine oil, icosapent ethyl (Vascepa), were invalidated by a federal district court judge, it came as a shock to Amarin Pharma, the company based in Ireland that had spent years and hundreds of millions of dollars developing its one and only pharmaceutical product. It was not surprising, therefore, that Amarin was prepared to file an appeal of the district court judge's opinion to the US Court of Appeals for the Federal Circuit, the court that hears many patent appeals.

Patents granted by the US Patent and Trademark Office (USPTO) to pharmaceutical companies on products they have developed are often of immense value. Simply put, a patent is a right bestowed on an inventor by the government to exclude competitors from making, using, selling or importing the invention without the express consent of the inventor. As pharmaceutical patents in the USA typically last 20 years, they may provide substantial protection to pharmaceutical companies from the threat of competition, allowing them the prospect of reaping large profits.

For a patent to be issued from the USPTO, specific criteria must be met. ${ }^{1}$ The invention must be patentable, useful and truly novel (35 U.S.C. $§ 102$ ), that is, it must not have been described in prior art, which includes previously issued patents, patent applications and in the case of drugs and other medical products, previously published research articles in the medical literature. Also, the invention must not be obvious (35 U.S.C. § 103). A patent is determined to be obvious (or nonobvious) on the basis of an examination of the prior art (ie, the information relevant to the product available at the time of the invention). If, before the date of patent filing, a person having ordinary skill in the art would have found the invention to be obvious based on the prior art (35 U.S.C. $\S 103$ ), then the patent should not be granted or should be invalidated if it was previously granted. Finally, the patentee must publicly disclose a written description of the invention and the best mode' of making it, so that a person having ordinary skill in the art may make and use the invention (35 U.S.C. § 112). Public disclosure is a critical part of the agreement between the patentee, who has been granted an important right, and society, which bestowed that right.

Vascepa was first approved by the US Food and Drug Administration (FDA) in July 2012, on the basis of the MARINE (Multi-center, plAcebo-controlled, Randomized, doubleblINd, 12-week study with an open-label Extension) clinical trial, ${ }^{2}$ for the indication of treatment of severe hypertriglyceridaemia $(\geq$ $500 \mathrm{mg} / \mathrm{dL}$ ). Subsequently, the manufacturer, Amarin, sponsored the ANCHOR (Effect of AMR101 (Ethyl Icosapentate) on TG Levels in Patients with High TG Levels) clinical trial, ${ }^{3}$ which provided evidence that Vascepa effectively lowered serum triglycerides in 
patients with baseline levels $\geq 200$ to $<500 \mathrm{mg} / \mathrm{dL}$. On the basis of this result, Amarin submitted a supplemental new drug application to the FDA to extend the label indication to include treatment of, in addition to triglyceride levels $\geq 500 \mathrm{mg} / \mathrm{dL}$, the lower range of triglyceride levels of $\geq 200$ to $<500 \mathrm{mg} / \mathrm{dL}$. The FDA rejected the application, stating that evidence had not been provided that treatment of lower levels of triglycerides would reduce the risk of cardiovascular disease.

Amarin and its legal team took the unusual step of filing a lawsuit against the FDA (Amarin Pharma, Inc. v. FDA), premised on Amarin's constitutional First Amendment right to promote truthful and non-misleading information derived from the ANCHOR trial to healthcare professionals. This right stems from the evolving jurisprudence on commercial speech, ${ }^{4}$ which is protected in the USA by the First Amendment (though not to the same extent as political speech). Federal Judge Paul Engelmayer of the Southern District of New York ordered Amarin and the FDA to reach a settlement, which affirmed Amarin's right to disseminate off-label promotional materials, including journal reprints, to healthcare professionals, as long as the materials were truthful and non-misleading. ${ }^{5}$ The judge's opinion spelled a significant legal victory for Amarin but which has stimulated considerable controversy. ${ }^{67}$ There are dangers inherent in allowing pharmaceutical companies to make claims for efficacy not reviewed or approved by regulators, and this practice may invite potential harm to public health. Still, the outcome of Amarin Pharma, Inc. v. FDA suggests that FDA may be limited to enforcing restrictions on off-label promotion only when the promotional activities are false and misleading. Off-label promotion that is shown to be truthful and non-misleading may elude FDA enforcement.

In December 2019, FDA granted Amarin a label extension for Vascepa to include reduction in risk of cardiovascular events in persons with serum triglyceride levels $\geq 150 \mathrm{mg} / \mathrm{dL}$, established cardiovascular disease or diabetes, and two or more additional risk factors for cardiovascular disease. The label extension was granted on the basis of the REDUCE-IT (Reduction of Cardiovascular Events with Icosapent Ethyl Intervention Trial) trial, ${ }^{8}$ a clinical trial in 8179 patients treated with maximal statin therapy who were randomised to receive iscosapent ethyl $4.0 \mathrm{~g} /$ day or mineral oil $4.0 \mathrm{~g} /$ day. After a median follow-up of 4.9 years, those treated with icosapent ethyl experienced a statistically and clinically significant $25 \%$ reduction in major adverse cardiovascular events (MACEs) as compared with the mineral oil comparison group. The label extension greatly extended the spectrum of patients who may qualify for treatment with Vascepa and also expanded Amarin's potential market for its product.

In the light of these developments, it is clear why Amarin was prepared to strongly appeal the district court judge's opinion that invalidated the six core patents on Vascepa. Invalidation of the patents stemmed from a lawsuit brought by Amarin against two generic drug companies,
Hikma Pharmaceuticals and Dr Reddy's Laboratories, both of which in 2016 had filed abbreviated new drug applications for their generic formulations of icosapent ethyl. Amarin's lawsuit against the companies (Amarin Pharma, Inc. v. Hikma Pharmaceuticals USA, Inc.) claimed that their proposed drug labels would induce physician prescribing behaviour in a manner that would infringe on Amarin's patents on Vascepa. Labels for generic drugs must be essentially identical to the labels for the associated innovator products, and in this light, Chief Judge Miranda Du of the US District Court for the District of Nevada ruled that the generic companies' labels would induce physicians to prescribe the generic products and thereby infringe on Amarin's patents. ${ }^{9}$ On the question of infringement, the generic companies lost their legal argument that their drug labels would not infringe Amarin's patents.

In the second part of her opinion, Judge Du turned to the question of obviousness. The generic companies argued that Amarin's patents on Vascepa were invalid because Vascepa's ability to lower serum triglycerides without raising low-density lipoprotein cholesterol (LDL-C) was obvious at the time the patents were issued. This claim was made on the basis of the prior art, principally an article by Mori et al. ${ }^{10}$ While marine oils often contain a combination of both eicosapentaenoic acid (EPA) and docosahexaenoic acid (DHA), Vascepa is a purified form of EPA, icosapent ethyl. Amarin's core patents on Vascepa all included claims that Vascepa lowers serum triglyceride levels without raising LDL-C levels, and this may not be the case with marine oils that contain a combination of EPA and DHA. In the study by Mori $e t a l,{ }^{10}$ the researchers compared the effects of purified EPA and purified DHA on lipid levels in mildly hyperlipidaemic men and concluded that DHA increased LDL-C while EPA did not. However, in reaching this conclusion, the authors made a common statistical error in failing to conduct a test for interaction between the changes in LDL-C associated with intake of purified EPA versus purified DHA. ${ }^{11} \mathrm{Had}$ they conducted that test, they would have discovered that there was no statistically significant difference between EPA and DHA in their effects on LDL-C levels. Judge Du, however, accepted the conclusion of Mori et $a l^{10}$ at face value and invalidated the Vascepa patents on the basis of an incorrect scientific conclusion in the prior art. ${ }^{9}$ Remarkably, a second published article that formed part of the prior art also contained a statistical error, which, if the judge had identified it, would have reversed her conclusion that lowering of apolipoprotein B by EPA was already demonstrated and therefore 'obvious'. ${ }^{12}$

On appeal to the US Federal Court of Appeals for the Federal Circuit, a three-judge panel affirmed the district court ruling for invalidation without even stating their reasons for the affirmation. The judges instead issued a one-word per curiam opinion the day after oral arguments: 'Affirmed'. ${ }^{13}$ It is clear that the court did not undertake a careful examination of the veracity of the prior art (Mori et $\left.a l^{10}\right)$ and did not consider the fundamental statistical 
error made by the authors in their study. Instead, it swiftly and uncritically affirmed the district court's error.

Errors such as these are not uncommon in issuing or declining to issue patents, as patent examiners at the USPTO face an increasingly unmanageable workload of patent applications. When examining prior art, it is generally acceptable practice that examiners may assume that the prior art is true and are under no obligation to undertake a deeper examination of the veracity of the prior art. As Freilich has pointed out, when they assess patent applications, examiners may be generally proficient in identifying the relevant prior art, but they do not often conduct peer review to determine the quality of the prior art. ${ }^{14}$ Freilich refers to this as proficiency in 'matching' but not in 'digging. ${ }^{14}$ While it may be unrealistic to expect USPTO patent examiners to carry out peer review of prior art, when patents reach the stage of litigation, court procedures, which probe the facts in greater depth, could include deeper examination of the quality of the prior art. In the case of the invalidation of Amarin's Vascepa patents, the district court judge and the expert witnesses for both the petitioner and defendant all failed to identify a fundamental statistical error in the prior art, and this error was left uncorrected by the Federal Circuit.

Indeed, the Federal Circuit has confirmed that district courts are under no obligation to probe the validity of prior art and may presume that the prior art, on discovery, is true. ${ }^{14}$ Freilich suggests that we should expect a more critical appraisal of the quality of prior art during patent litigation in the courts than during patent prosecution by the USPTO ${ }^{14}$ However, this did not take place in the Vascepa patent litigation, at either the trial or appellate levels. Amarin appealed further and requested en banc review by the full Federal Circuit, but the court denied the appeal.

Meanwhile, in May 2020, the FDA approved the first generic formulation of icosapent ethyl, produced by Hikma Pharmaceuticals USA (one of the defendants in the Vascepa patent case). With FDA approval in hand and invalidation of the six core Vascepa patents having been affirmed by the Federal Circuit, Hikma is now positioned to launch its generic equivalent into the US market. The generic formulation will provide a lower cost alternative to patients while also competing with Amarin's Vascepa. The US market for Vascepa will undoubtedly be diminished.

Vascepa's story has been further complicated by the recent publication of the STRENGTH (Long-Term Outcomes Study to Assess STatin Residual Risk Reduction With EpaNova in HiGh CV Risk PatienTs With Hypertriglyceridemia) trial. ${ }^{15}$ STRENGTH was a randomised, double-blind clinical trial of an omega-3 fatty acid preparation versus corn oil in 13078 statin-treated patients at high cardiovascular risk. The omega-3 preparation was a carboxylic acid (CA) preparation, which may increase absorption, and consisted of a mixture of EPA and DHA at a total dose of $4.0 \mathrm{~g} /$ day (Epanova). The comparator was corn oil at a dose of $4.0 \mathrm{~g} /$ day. The total daily dose of the EPA and DHA combination was comparable to the total dose of omega-3 fatty acids used in the REDUCE-IT trial, although an important difference was that purified EPA was used in REDUCE-IT. The STRENGTH trial was terminated prematurely after a median of 39 months of follow-up because of futility. The frequency of the primary end point, MACEs, was virtually identical at $12 \%$ in both randomised groups. Thus, taken at face value, the null results of STRENGTH directly contradict the positive results of REDUCE-IT, which found a significant benefit of EPA on MACEs compared with mineral oil.

What might explain the discrepant results of these two important clinical trials? One possibility is that the omega-3 preparations were not identical. While REDUCE-IT employed a purified preparation of EPA (icosapent ethyl $4.0 \mathrm{~g}$ /day), STRENGTH employed a CA preparation that contained both EPA and DHA (a total of $4.0 \mathrm{~g} /$ day). Calder and Deckelbaum ${ }^{16}$ astutely pointed out that the actual EPA content of Vascepa is $960 \mathrm{mg}$ per capsule or $3.84 \mathrm{~g}$ per four capsules, while Epanova consists of EPA, $550 \mathrm{mg}$ per capsule or $2.2 \mathrm{~g}$ per four capsules, and DHA, $200 \mathrm{mg}$ per capsule or $0.8 \mathrm{~g}$ per four capsules. The different doses of EPA were also reflected in different serum and plasma levels of EPA in the two trials. ${ }^{815}$ Therefore, it is possible that the different clinical outcomes in REDUCE-IT and STRENGTH may be explained by the different doses of EPA used in the two trials. Although it is conceivable that DHA may increase the risk of cardiovascular events, it would be an unlikely coincidence that DHA exactly offset a beneficial effect of EPA, resulting in a null outcome in STRENGTH. It is also noteworthy that in REDUCE-IT, clinical benefit was associated with achieved EPA serum levels but not with changes in serum triglyceride levels. ${ }^{17}$

Another possibility is that the mineral oil comparator in REDUCE-IT had an adverse effect on cardiovascular risk, rather than EPA having a beneficial effect. In support of this possibility were the findings in REDUCE-IT that the administration of mineral oil was associated with increases in LDL-C (7.4\%), apolipoprotein B $(6.7 \%)$ and high-sensitivity C-reactive protein $(37.6 \%)$, at last visit, relative to icosapent ethyl. ${ }^{8}$ Each of these biomarker changes may be associated with an increase in risk of cardiovascular events. These biomarker changes in response to mineral oil are not unique to REDUCE-IT, and similar biomarker increases were observed in both the MARINE ${ }^{2}$ and the ANCHOR ${ }^{3}$ trials, both of which also compared icosapent ethyl with mineral oil 'placebo'. The principal outcome data in these trials were placebo-adjusted and therefore may have appeared to be greater than the actual values. For example, in ANCHOR the placebo-adjusted change in LDL-C with icosapent ethyl $(4.0 \mathrm{~g} /$ day $)$ was $-6.2 \%$. Without placebo adjustment, the value was $+1.5 \%$, indicating that the apparent reduction in LDL-C with icosapent ethyl was fully explained by the increase in LDL-C with mineral oil. Nevertheless, when the REDUCE-IT data were reviewed by the FDA (and also independently 
by Health Canada, the European Medicines Agency and the Committee for Medicinal Products for Human Use), the FDA reviewers concluded that the increase in levels of the biomarkers with mineral oil would explain only a small percentage of the $25 \%$ difference in MACEs between the two randomised groups (https://www.fda. gov/media/132479/download). This conclusion was based on a review of the literature on mineral oil and post hoc analyses of data from REDUCE-IT. An independent review article on mineral oil was also recently published. ${ }^{18}$ It is noteworthy that increases in the levels of these three biomarkers were not observed in the corn oil comparison group in STRENGTH. ${ }^{15}$

A further consideration is the JELIS (Japan EPA Lipid Intervention Study) trial, in which 18645 patients were randomised (open label) to receive $1.8 \mathrm{~g}$ of EPA per day plus statin therapy or statin therapy alone. ${ }^{19}$ After a mean of 4.6 years, there was a statistically significant $19 \%$ reduction in MACEs in the EPA/statin group as compared with the statin-alone group. Thus, with a purified preparation of EPA at nearly the same dose used in STRENGTH, but without DHA or placebo mineral oil, MACEs were significantly reduced, in contrast with the findings in STRENGTH. However, it should be noted that the JELIS trial was not conducted according to contemporary standards of care. Mean baseline LDL-C levels were $180 \mathrm{mg} / \mathrm{dL}$, and patients were treated with an average dose of only $10 \mathrm{mg}$ of pravastatin or $5 \mathrm{mg}$ of simvastatin, which would be considered to be suboptimal statin therapy today. A secondary prevention trial of EPA plus statin therapy (RESPECT-EPA) is in progress. ${ }^{20}$

We are left with uncertainty about the explanation for the discrepant results among these clinical trials. ${ }^{21-23}$ Possible biological and mechanistic explanations have been recently discussed. ${ }^{24}$ The STRENGTH trial raises the question of whether Vascepa actually does reduce the risk of cardiovascular events in patients at risk for them. A direct comparison of icosapent ethyl with corn oil in a new randomised clinical trial would settle the matter, but it seems unlikely that Amarin would undertake such an expensive trial, especially in the light of the invalidation of its patents and probable diminution of the US market for Vascepa. Still, patients and physicians need to know whether icosapent ethyl has cardiovascular health benefits, and FDA should mandate a clinical trial to answer the question.

The story of Vascepa, which is still unfolding, is a telling example of the intersection between drug development and the law. In this circumstance, the Irish company Amarin prevailed in an important lawsuit against the FDA involving off-label promotion of Vascepa, a case that has had significant ramifications for First Amendment coverage of commercial free speech. Success in that lawsuit, however, was followed by a major setback in a separate lawsuit resulting in invalidation of Amarin's core patents on Vascepa. The patent invalidation, along with the results of the STRENGTH trial, which call into question the very efficacy of the drug, has placed the future of this product in considerable jeopardy. We await the next chapter in this interesting, evolving tale.

Contributors GC wrote the first draft of the manuscript and serves as the corresponding author. ES edited the manuscript and provided valuable intellectual input.

Funding The authors have not declared a specific grant for this research from any funding agency in the public, commercial or not-for-profit sectors.

Competing interests None declared.

Patient consent for publication Not required.

Provenance and peer review Not commissioned; externally peer reviewed.

Data availability statement This article contains no new data. All the data referred to are already in the published literature and are fully available.

Open access This is an open access article distributed in accordance with the Creative Commons Attribution Non Commercial (CC BY-NC 4.0) license, which permits others to distribute, remix, adapt, build upon this work non-commercially, and license their derivative works on different terms, provided the original work is properly cited, appropriate credit is given, any changes made indicated, and the use is non-commercial. See: http://creativecommons.org/licenses/by-nc/4.0/.

ORCID iD

Gregory Curfman http://orcid.org/0000-0002-9157-9585

\section{REFERENCES}

1 Field TL. Obviousness as fact: the issue of Obviousness in patent law should be a question of fact reviewed with appropriate Deference, 27 Fordham Intell. Prop Media \& Ent LJ 2017;555.

2 Bays HE, Ballantyne CM, Kastelein JJ, et al. Eicosapentaenoic acid ethyl ester (AMR101) therapy in patients with very high triglyceride levels (from the Multi-center, plAcebo-controlled, Randomized, double-bllNd, 12-week study with an open-label Extension [MARINE] trial). Am J Cardiol 2011;108:682-90.

3 Ballantyne CM, Bays HE, Kastelein JJ. A phase 3, multicenter, placebo-controlled, randomized, double-blind, 12-week study to evaluate the effect of two doses of AMR101 on fasting serum triglycerides and other lipid parameters in statin-treated patients with persistent high triglycerides ( $\geq 200$ and. Am J Cardiol 2012;110:984-92.

4 Post R. The constitutional status of commercial speech. 48 UCLA L. Rev. 1 (2000-2001).

5 Mazer D, Curfman GD. Fda sanctions off-label drug promotion. Health Affairs Blog.

6 Amarin Pharma, Inc., v. FDA. Harvard law review. 2016; 129 (NO. 7). Available: https://harvardlawreview.org/2016/05/amarin-pharma-inc$\mathrm{v}$-fda/

7 Bragg JL. Amarin settlement erodes off-label promotion enforcement. Law360, 2016. Available: https://www-law360-com. ezproxy.neu.edu/articles/770109/amarin-settlement-erodes-offlabel-promotion-enforcement

8 Bhatt DL, Steg PG, Miller M, et al. Cardiovascular risk reduction with icosapent ethyl for hypertriglyceridemia. N Engl J Med 2019;380:11-22.

9 Du MM. United States district Court District of Nevada. Case 2:16-cV-02525-MMD-NJK. document 3812020.

10 Mori TA, Burke V, Puddey IB, et al. Purified eicosapentaenoic and docosahexaenoic acids have differential effects on serum lipids and lipoproteins, LDL particle size, glucose, and insulin in mildly hyperlipidemic men. Am J Clin Nutr 2000;71:1085-94.

11 Curfman G, Bhatt DL, Pencina M. Federal judge invalidates icosapent ethyl patents - but on the basis of a common statistical mistake. Nat Biotechnol 2020;38:939-41.

12 Kurabayashi T, Okada M, Tanaka K. Eicosapentaenoic acid effect on hyperlipidemia in menopausal Japanese women. The Niigata Epadel Study Group. Obstet Gynecol 2000;96:521-8.

13 United States Court of Appeals for the Federal Circuit. Amarin pharma Inc. V Hikma pharmaceuticals USA, Inc. case 20-1723. document 78. Filed 09/03/ 2020.

14 Freilich J. Matching and digging: evidentiary analysis at the patent office. Fordham Law ReviewIn press.

15 Nicholls S, Lincoff S, Garcia M. A randomized clinical trial comparing high-dose omega-3 fatty acids to corn oil placebo on major adverse cardiovascular events. JAMA2020. 
16 Calder PC, Deckelbaum RJ. Editorial: omega-3 fatty acids: new studies, new data, new questions. Curr Opin Clin Nutr Metab Care 2021;24:109-13.

17 Bhatt DL, Miller M, Steg PG. Achieved eicosapentaenoic acid levels strongly predict cardiovascular benefit in REDUCE-IT. presented at the 2020 American College of Cardiology/World Congress of cardiologyAbstract 20-LB-20501-ACC.

18 Olshansky B, Chung MK, Budoff MJ, et al. Mineral oil: safety and use as placebo in REDUCE-IT and other clinical studies. Eur Heart $J$ Supp/ 2020;22:J34-48.

19 Yokoyama M, Origasa $\mathrm{H}$, Matsuzaki M, et al. Effects of eicosapentaenoic acid on major coronary events in hypercholesterolaemic patients (JELIS): a randomised open-label, blinded endpoint analysis. Lancet 2007;369:1090-8.

20 . Available: https://urldefense.proofpoint.com/v2/url?u=https-3A upload.umin.ac.jp_cgi-2Dopen-2Dbin_ctr-5Fe_ctr-5Fview.cgi3Frecptno-3DR000014051\&d=DwIFaQ\&c=iqeSLYkBTKTEV8nJYtdW
A\&r=d8j9rSy3wS5egb8Bq5puQU7zqAjJzrclomzRjA h8dY\&m= vFDTc8SgePYGbIZh1LI-GtAJMNqJVb5PJAU0EcFwREE\&s=fFC2 vH1byrwvVbdB6dgvUd6JW6zu26m4QUUC8wr_c3E\&e=

21 Sharma G, Martin SS, Blumenthal RS. Effects of omega-3 fatty acids on major adverse cardiovascular events: what matters most: the drug, the dose, or the placebo? JAMA 2020;324:2262-4.

22 Curfman G. Do omega-3 fatty acids benefit health? JAMA 2020;324:2280-1.

23 Volpe M, Patrono C. The REDUCE-IT verdict on eicosapentaenoic acid and cardiovascular outcome challenged with strength. Eur Heart J 2021;42:370-1.

24 Mason RP, Libby P, Bhatt DL. Emerging mechanisms of cardiovascular protection for the omega-3 fatty acid eicosapentaenoic acid. Arterioscler Thromb Vasc Biol 2020:40:1135-47. 